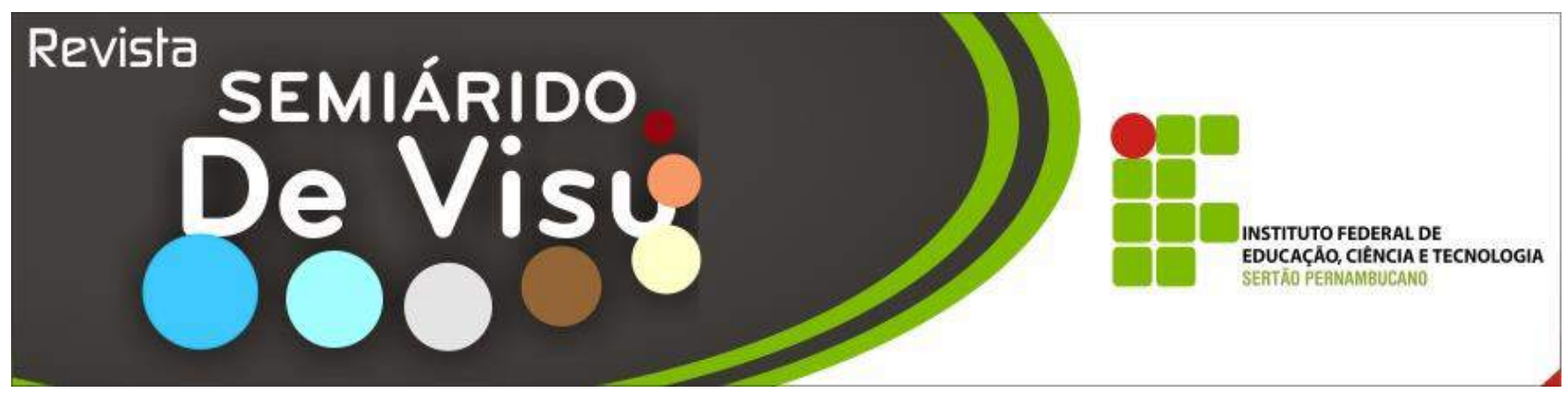

\title{
Economias em desenvolvimento e o Consumo de Frutas e Hortaliças
}

\author{
Rosemary Barbosa de Melo ${ }^{1}$; Reney Dorow ${ }^{2}$; Caroline Pauletto Spanhol Finocchio ${ }^{3}$; Thiago Borges Feitosa ${ }^{4}$
}

\author{
${ }^{1}$ Instituto Federal de Educação Ciência e Tecnologia do Sertão Pernambucano - Campus Petrolina Zona Rural \\ Rodovia BR 235, Km 22, Projeto Senador Nilo Coelho - N4, CEP: 56300-000 | Petrolina/PE - Brasil, Fone: (87) 2101-8050 - E- \\ mail: rosemarybm1@hotmail.com; \\ ${ }^{2}$ Epagri - Empresa de Pesquisa Agropecuária e Extensão Rural de Santa Catarina, Rodovia Admar Gonzaga, 1.347, Itacorubi, Caixa \\ Postal 502 88034-901 Florianópolis, SC, Brasil Fone: (48)3665-5000, fax: (48)3665-5010; \\ ${ }^{3}$ Universidade Federal do Mato Grosso do Sul, Rua Costa e Silva S/N, Cidade Universitária CEP: 79070-900 Campo Grande , Fone: \\ (67) 3345-7001; \\ ${ }^{4}$ Universidade Federal do Vale do São Francisco, Av. José de Sá Maniçoba, s/n - Centro CEP 56304-917, Petrolina-PE Fone: (87) \\ 21016705 , Fax:(87) 21016830 ;
}

\begin{abstract}
RESUMO: O Objetivo é analisar o consumo de Frutas e Hortaliças (F\&H) no Brasil, verificar a influência da renda nesse consumo e identificar ações de intervenção para a obesidade através do estímulo ao seu consumo. Foram utilizados os dados de consumo de frutas e hortaliças, de renda e os referentes à prevalência do excesso de peso e obesidade disponíveis nos relatórios da Pesquisa de Orçamentos Familiares nos anos de 2002-03/ 2008-09, da pesquisa da Confederação Nacional da Agricultura e da Vigilância de Fatores de Risco e Proteção para Doenças Crônicas por Inquérito Telefônico. Realizou-se comparações de consumo de F\&H entre as grandes regiões brasileiras, utilizando o teste estatístico Kruskall-Wallis para verificar se havia diferenças significativas entre elas com relação ao consumo médio desses produtos. Para analisar a influência da renda no consumo de F\&H foi utilizada a Correlação de Pearson. Concluiuse que o consumo de F\&H no Brasil está abaixo do recomendado pela Organização Mundial de Saúde e que a renda exerce influência no consumo desses alimentos.
\end{abstract}

Palavras-chave: Alimentação saudável, renda, obesidade, consumo, agronegócio.

\section{Developing economies and consumption of fruits and vegetables}

\begin{abstract}
To analyze the consumption of fruits and vegetables $(\mathrm{F} \& \mathrm{~V})$ in Brazil, verify the influence of the income on that consumption, and identify interventions for obesity by encouraging consumption. The data used were those for fruit and vegetable consumption, income and those referring to the prevalence of overweight and obesity available from the reports on Research on Family Budgets in the years 2002-03/2008-09, da research by the National Confederation of Agriculture and those by the Surveillance of Risk Factors and Protection for Chronic Diseases by Phone Questioning . Comparisons were made between the consumption of F\&V in the major Brazilian regions, using the statistical KruskallWallis test to verify whether there were significant differences between them with respect to the average consumption of these products. In order to analyze the influence of income on consumption of F\&V the Pearson Correlation Coefficient was used. . It was observed that the $\mathrm{F} \& \mathrm{~V}$ consumption in Brazil is below the recommended by the World Health Organization (WHO). It was also possible to verify that income has influence on the consumption of these foods.
\end{abstract}

Keywords: Healthy eating, obesity, income, consumption, agribusiness. 


\section{Introdução}

$\mathrm{N}$ as últimas décadas, condições favoráveis à ocorrência de deficiências nutricionais, têm sido substituídas por epidemia de obesidade e doenças crônicas nãotransmissíveis $^{1}$ relacionadas ao consumo excessivo e desequilibrado de alimentos. No Brasil, o déficit de altura entre crianças declinou da faixa dos $30 \%$ para menos de $10 \%$, isso nos últimos 35 anos. Atualmente o déficit de peso atinge hoje menos de $5 \%$ da população, por outro lado o sobrepeso e a obesidade ${ }^{2}$ vem crescendo no País. Além disso, observou-se que no período 2006-2010 houve um aumento de excesso de peso $^{3}$ em 1,2 ponto percentual ao ano entre os homens, enquanto entre as mulheres, esse aumento foi de 2,2 ponto percentual ao ano. O número de obesos em ambos os sexos, variou de $12,7 \%$ para 13,9\% no período de 2003 a 2009 (BRASIL, 2010a). O excesso de peso e a obesidade entre jovens e crianças também têm sido preocupantes. A avaliação do estado nutricional de crianças de 5 a 9 anos de idade, estudada pela POF 2008-09, mostrou que o excesso de peso e a obesidade já atingem 33,5\% e 14,3\%, respectivamente (IBGE, 2010a).

A causa da obesidade é multifatorial, ou seja, ela é decorrente de vários fatores, tais como hábitos de vida, características socioambientais e susceptibilidade genética/biológica (WHO, 2011b) que podem estar agindo isoladamente ou em conjunto. Entre esses fatores, está o baixo consumo de alimentos saudáveis, tais como as frutas e hortaliças. A recomendação mínima de consumo dos mesmos é de $400 \mathrm{~g} / \mathrm{dia}$, o que corresponde no Brasil de $6 \%$ a $7 \%$ das calorias totais de uma dieta de $2.300 \mathrm{kcal}$ diárias (FAO/WHO/ONU, 2004). Isso de acordo com o

\footnotetext{
1 DCNT( circulatórias, câncer, respiratórias crônicas e diabetes) e seus fatores de risco em comum modificáveis (tabagismo, álcool, inatividade física, alimentação não saudável e obesidade).

2 A obesidade é uma doença caracterizada por um excessivo acúmulo de gordura nos tecidos. Trata-se de um distúrbio que, além dos problemas de natureza estética e psicológica, constitui risco para a saúde. A obesidade pode ser calculadas pelo índice de massa corporal (IMC), pelo
}

Guia Alimentar para a População Brasileira (BRASIL, 2005).

O baixo consumo de frutas e hortaliças tem sido associado ao risco de ocorrência de certos tipos de câncer e doenças cardiovasculares (LIU et al., 2000; RIBOLE \& NORAT, 2003). Esse déficit de consumo representa um dos dez fatores centrais da carga global de obesidade (WHO, 2002). Dada a importância do consumo de frutas e hortaliças na intervenção para a obesidade, o presente estudo teve por objetivo analisar o consumo desses produtos no Brasil, verificar a influência da renda neste consumo e identificar ações de estímulo ao consumo dos mesmos nas dimensões micro e macroambiental, tendo em vista que, o aumento da sua ingestão é benéfica tanto para a saúde da população quanto para o crescimento das vendas de $\mathrm{F} \& \mathrm{H}$ o que poderá impactar positivamente o setor frutícola e olerícola.

\section{Ambiente obesogênico e a ação benéfica do consumo de frutas e hortaliças}

$\mathrm{O}$ ambiente obesogênico diz respeito à influência que oportunidades e condições ambientais têm nas escolhas, por parte dos indivíduos, de hábitos de vida que promovam o desenvolvimento da obesidade. $\mathrm{O}$ ambiente seria representado pelos ambientes físico, econômico e sociocultural no qual o indivíduo está inserido (EGGER \& SWINBURN, 1997). Desse modo, o estilo de vida de cada indivíduo é fortemente influenciado pelo ambiente. Atualmente o ambiente em que vivemos é considerado obesogênico, isto é, ambiente propício à obesidade, pois seduz e induz à

cálculo do peso/(altura) ${ }^{2}$, que mede o nível de corpulência. Segundo a OMS há obesidade quando o índice é $\geq$ a 30 . O diagnóstico de obesidade em crianças e adolescentes não é aplicável de modo igual ao dos adultos, devido às características dinâmicas dos processos de crescimento e de maturação que ocorrem durante o desenvolvimento infantil.

${ }^{3}$ Excesso de peso é diagnosticado quando o IMC é $\geq 25$ $\mathrm{kg} / \mathrm{m} 2 \mathrm{e}<30 \mathrm{~kg} / \mathrm{m} 2$ (OMS,2002). 
adoção de comportamentos alimentares inadequados e sedentarismo (CYPRESS, 2004). Assim, um ambiente obesogênico favorece a adoção de comportamentos obesogênicos, como por exemplo, consumo de alimentos com alta densidade energética (BOUCHARD, 2007).

Entender o papel do ambiente no aumento da prevalência da obesidade é, portanto, fundamental para desenvolver estratégias efetivas de prevenção dessa doença e, assim, reduzir seus impactos negativos na saúde das populações. A obesidade aumenta em grande escala o risco de aparecimento, desenvolvimento e agravamento de outras doenças. Por essa razão a Organização Mundial de Saúde - OMS (2011) a considera como uma doença epidêmica global do século 21. Estimase que essa doença mata quase 3 milhões de pessoas por ano no mundo e que $12 \%$ da população mundial é considerada obesa. No continente americano $26 \%$ dos adultos são obesos, sendo a região com maior incidência do problema. No extremo oposto está o Sudeste Asiático, com apenas 3\% de obesos. Em todas as regiões do mundo, a obesidade duplicou entre 1980 e 2008. A projeção para 2015 é que haverá 700 milhões de obesos no mundo, indicando um aumento de 75\% em 10 anos (WHO, 2011a). Um dos principais fatores de risco para obesidade é a alimentação não saudável, por isso está sendo considerada a mais importante desordem nutricional, tanto nos países desenvolvidos como também nos países em desenvolvimento, devido ao aumento de sua incidência (POPKIN \& DOAK, 1998; MALTA et al., 2007). Visando melhorar a qualidade da alimentação no mundo, a Organização Mundial de Saúde - OMS, estabeleceu uma Estratégia Global, que estimula o consumo de alimentos saudáveis, dentre eles, o de frutas e hortaliças $\mathrm{F} \& \mathrm{H}$. O baixo consumo desses alimentos encontra-se no ranking dos dez principais fatores de risco de mortalidade global. Há evidências científicas de que o consumo de F\&H pode proteger o organismo contra déficits de vitaminas e minerais, aumentar a resistência contra infecções e auxiliar na prevenção do câncer gastrointestinal e infartos. Estima-se que a baixa ingestão desses alimentos cause $10 \%$ do câncer gastrointestinal, $31 \%$ das doenças do coração e $11 \%$ dos infartos no mundo. Doenças que na maioria dos casos são associadas à obesidade (BRASIL, 2011b).

\section{Material e métodos}

A pesquisa envolveu coleta de dados primários e secundários. Na etapa de coleta de dados secundários foi realizada uma pesquisa bibliográfica em livros e artigos que tratam da temática abordada. Além disso, foram consultados dados disponibilizados pelo Instituto Brasileiro de Geografia e Estatística (IBGE) - Estudo Nacional de Despesa Familiar (ENDEF) 1974-75; Instituto Nacional de Alimentação e Nutrição 1989 e Pesquisa de Orçamentos Familiares (POF) 2002-03/200809. Cabe ressaltar que tais dados apresentam a limitação de serem autorreferidos. Logo, estão sujeitos a um viés de informação (SCHMIDT et al., 2011). Também foram consultados dados da Pesquisa Quantitativa e Qualitativa das Frutas, Legumes e Hortaliças, disponibilizada, em 2011, pela Confederação Nacional da Agricultura -CNA e da Vigitel Brasil 2010 (Vigilância de Fatores de Risco e Proteção para Doenças Crônicas por Inquérito Telefônico). Quanto às fontes primárias, foi realizada uma entrevista com uma profissional da nutrição para a obtenção de informações sobre o consumo de frutas e hortaliças, bem como sobre a prevalência do excesso de peso e obesidade no Brasil. Buscou-se, ainda, identificar a percepção da profissional quanto às ações existentes para se estimular o consumo de frutas e hortaliças no Brasil. Para a interpretação de dados foram utilizadas análises descritivas sobre o consumo de frutas e hortaliças, tais como a média e a frequência de consumo. Nessa etapa da análise foi utilizado o software Excel. Para a análise da entrevista, foi aplicada a técnica de análise de conteúdo (BARDIN, 1977). Foram realizadas comparações de consumo de F\&H entre as grandes regiões brasileiras, utilizando o teste estatístico Kruskall-Wallis para verificar se havia diferenças significativas entre elas com relação ao consumo médio desses produtos. E, para analisar a influência da renda no consumo de F\&H foi utilizada a Correlação de Pearson. 
Ressalta-se que as análises inferenciais realizadas foram em relação aos dados disponíveis nas fontes acima citadas.

\section{Resultados}

Prevalências de excesso de peso e obesidade no Brasil

A Organização para a Cooperação e Desenvolvimento Econômico - OCDE (2011), recomenda aos países em desenvolvimento que adotem medidas imediatas para conter $o$ crescente número de pessoas obesas, antes que essas nações alcancem índices semelhantes aos registrados em países ricos. O Brasil é um dos países com índices preocupantes de obesidade. A evolução da obesidade no Brasil é atrelada aos maus hábitos alimentares da população Brasileira e ao sedentarismo (BRASIL, 2011b). Pode-se observar na Figura 1 que o problema da obesidade no Brasil atinge a todas as faixas etárias, tanto no sexo masculino como no feminino. A figura também apresenta dados de excesso de peso, pois este é considerado pelos estudiosos como um estado de alerta.

Figura 1 - Evolução de indicadores antropométricos de excesso de peso e obesidade na população por faixa etária (\%) e por sexo. Fonte: Estudo Nacional de Despesa Familiar (ENDEF) 1974-1975; Instituto Nacional de Alimentação e Nutrição 1989 e Pesquisa de Orçamentos Familiares (POF) 2002-2003/2008-2009. IBGE. Antropometria e Estado Nutricional de Crianças, Adolescentes e Adultos no Brasil, 2010.

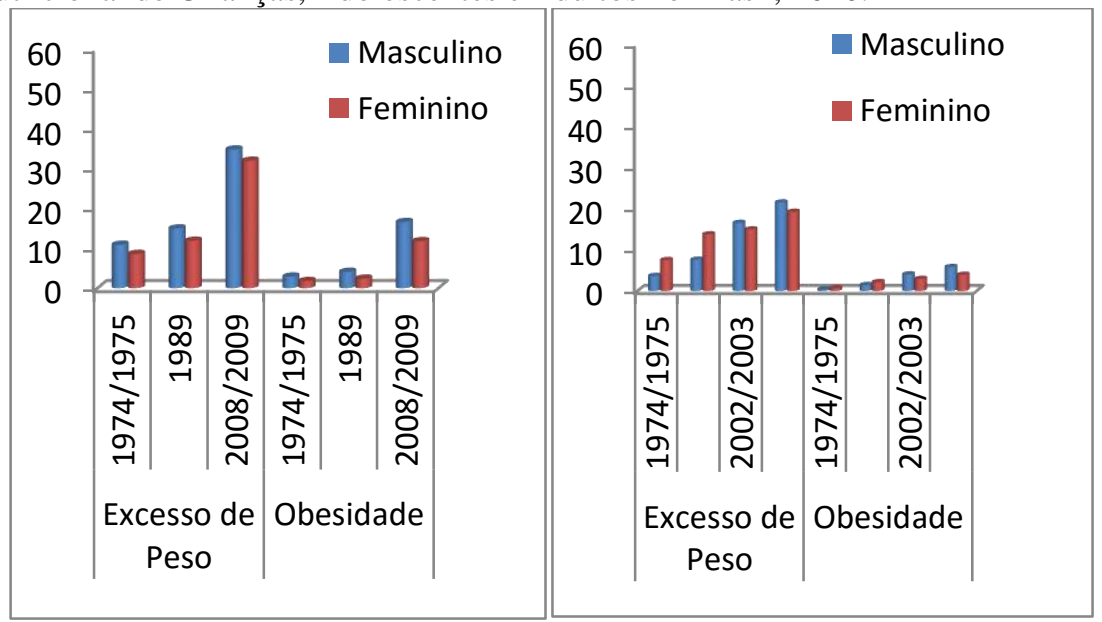

(a) 5 a 9

(b) 10 a 19

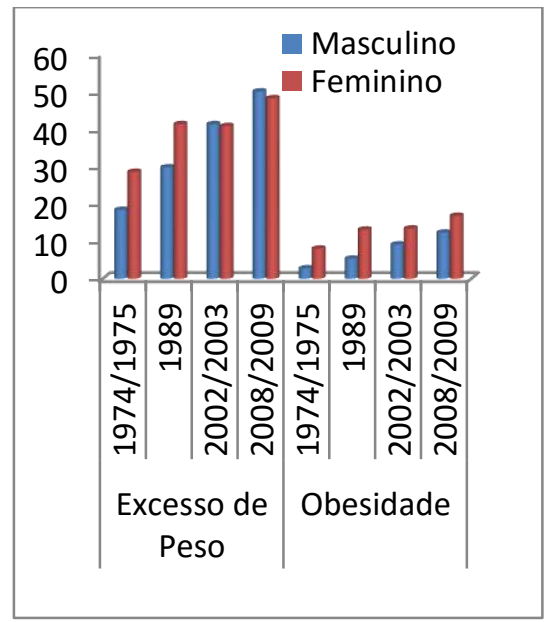

(c) 20 ou mais

Fonte: (IBGE, 2010).

No período de 2008-09, o excesso de peso foi observado em $33,5 \%$ das crianças entre 5 a 9 anos, sendo que $16,6 \%$ dos meninos também eram obesos; entre as meninas, a obesidade apareceu em 11,8\%. Esses números representam um salto na frequência de excesso de peso nessa faixa etária ao longo de 34 anos em meninos: 34,8\% em 2008-09, 15\% em 1989 e 10,9\% em 1974-75. Observou-se padrão semelhante nas meninas: $32 \%$ em 2008-09, 11,9\% em 1989 e 8,6\% em 1974-75 (Figura 1a). A região brasileira com maior frequência de excesso de peso foi a Sudeste, com 40,3\% dos meninos e $38 \%$ das meninas com peso acima do normal (IBGE, 2010a). Entre os indivíduos do sexo masculino entre 10 a 19 anos de idade, a frequência de excesso de peso passou de 3,7\% (1974-75) para 21,7\% (200809); já no sexo feminino, o crescimento do excesso de peso foi de $7,6 \%$ para $19,4 \%$ na mesma faixa etária. A obesidade também apresenta tendência ascendente, indo de 0,4\% para $5,9 \%$ no sexo masculino e de $0,7 \%$ para $4,0 \%$ no sexo feminino, comparando os mesmos períodos (Figura 1b). Apesar de ocorrer em todas as regiões brasileiras, a Região Sul tem o maior crescimento na frequência de excesso de peso, tendo sido encontradas variações de $4,7 \%$ para $27,2 \%$ nos adolescentes e $9,7 \%$ para $22,0 \%$ nas 
adolescentes (IBGE, 2010a). A Pesquisa de Orçamentos Familiares (POF) 2008-09 mostra um aumento contínuo de excesso de peso e obesidade na população com mais de 20 anos de idade ao longo de 35 anos. O excesso de peso quase triplicou entre homens, de $18,5 \%$ em 1974-75 para 50,1\% em 2008-09. Nas mulheres, o aumento foi menor: de $28,7 \%$ para $48 \%$. Já a obesidade cresceu mais de quatro vezes entre os homens, de $2,8 \%$ para $12,4 \%$ ( $1 / 4$ dos casos de excesso) e mais de duas vezes entre as mulheres, de $8 \%$ para $16,9 \%$. Também entre adultos a Região Sul foi a que apresentou as maiores frequências, tanto de excesso de peso $(56,8 \%$ de homens, $51,6 \%$ de mulheres), quanto de obesidade: $15,9 \%$ e homens e $19,6 \%$ de mulheres (IBGE, 2010a).

O número de obesos no Brasil cresceu de 11,4\% para 13,9\% no período de 2006 a 2009 (BRASIL, 2011b). Junto com o aumento da obesidade no País, a realização de procedimentos de redução de estômago - denominadas cirurgias bariátricas ${ }^{4}$ - tem registrado um crescimento exponencial, com um aumento de $275 \%$ nos últimos sete anos. O número de procedimentos subiu de 16 mil, em 2003, para 60 mil, em 2010. O País fica atrás apenas dos Estados Unidos no ranking mundial, os americanos estão na frente, com cerca de 300 mil cirurgias do tipo realizadas em 2010 (SBCBM, 2012).

\section{Consumo de frutas e hortaliças do Brasil}

Como já relatado, o consumo diário recomendado de F\&H é de no mínimo $400 \mathrm{~g}$, o que corresponde a $146 \mathrm{~kg}$ por ano no Brasil. Com o intuito de identificar se há diferença no consumo desses produtos entre as Regiões do Brasil, foi aplicado o Teste Kruskal-Wallis, o qual comparou o consumo médio de F\&H entre as regiões brasileiras, com base nos dados da POF $^{5}$ 2002-03 e 2008-09, cujos resultados estão na Tabela 1.

Tabela 1 - Ranking de consumo de frutas e hortaliças para as Regiões Brasileira, segundo Kruskal-Wallis para 1\% de probabilidade referente ao período 2002-2003 e 2008-2009.

\begin{tabular}{c|c|c}
\multicolumn{2}{c|}{$\begin{array}{c}\text { The GLM Procedure } \\
\text { Least Squares Means }\end{array}$} & $\begin{array}{c}\text { Média de } \\
\text { consumo por } \\
\text { região para os } \\
\text { dois períodos } \\
\text { avaliados (kg per } \\
\text { capita) }\end{array}$ \\
\hline Norte & $\begin{array}{c}\text { reast-squares } \\
\text { means }\end{array}$ & 36,85 \\
\hline Nordeste & 15.0357143 & 44,52 \\
\hline Centro-Oeste & 23.6111111 & 47,76 \\
\hline Sudeste & 39.6250000 & 56,78 \\
\hline Sul & 50.1666667 & 72,77 \\
\hline \multicolumn{3}{|c|}{} \\
\hline
\end{tabular}

Face ao exposto acima se entende que tanto no período 2002-03 quanto em 2008-09 houve diferenças significativas entre os consumos de $\mathrm{F} \& \mathrm{H}$ em relação às regiões brasileiras. Verificou-se através da Tabela 1 que a Região Sul se destaca no consumo do grupo

\footnotetext{
${ }^{4}$ Corresponde a redução do estômago para perda de peso sendo recomendada quando o índice de massa corporal (IMC) é maior que $40 \mathrm{~kg} / \mathrm{m}^{2}$ em pessoas com idade superior a 18 anos, seja homem ou mulher. O procedimento pode ser comentado, ainda, se o IMC estiver entre $35 \mathrm{~kg} / \mathrm{m}^{2}$ e $40 \mathrm{~kg} / \mathrm{m}^{2}$ e o paciente em questão
}

F\&H, mesmo assim, está distante do recomendado pela OMS. A Região Norte apresenta o menor consumo. A diferença entre estes extremos é de aproximadamente $97,47 \%$. $\mathrm{Na}$ Região Norte o estado que se destacou em média, no período de 2002-03 e 2008 -09, no

tiver diabetes, hipertensão arterial, apneia do sono, hérnia de disco ou outras doenças associadas à obesidade.

${ }^{5}$ Os resultados apresentados pela Pesquisa de Orçamento Familiar referem-se às estimativas das quantidades de alimentos adquiridos para consumo no domicílio, no ano e per capita, obtidas no período de 2008-2009. 
consumo do grupo $\mathrm{F} \& \mathrm{H}$ foi Rondônia com 54 $\mathrm{kg} / \mathrm{ano}$, no Nordeste foi Pernambuco com 56 $\mathrm{kg} / \mathrm{ano}$, no Sudeste foi o Rio de Janeiro com $61 \mathrm{~kg} / \mathrm{ano}$, no Sul foi o Rio Grande do Sul com $81 \mathrm{~kg} / \mathrm{ano}$ e no Centro-Oeste é o Distrito Federal com 56kg/ano (IBGE, 2004; 2010a). Quanto a aquisição domiciliar per capita média de frutas, no Brasil, em 2002-03 foi 24,487 kg e em 200809 o consumo médio foi de $28,863 \mathrm{~kg}$ (aumento de 17,9\%). Quanto as Hortaliças, em 2002-03, o consumo foi de $29,002 \mathrm{~kg}$ e em 2008-09 essa média foi de $27,075 \mathrm{~kg}$ (redução de 6,7\%). Referente às despesas verificou-se que a média mensal familiar ainda é pequena em relação a outros gêneros alimentícios, sendo de 4,6\% para frutas e 3,3\% para legumes e verduras (IBGE, 2010b). Conforme a POF 2008-09, apenas 10\% da população brasileira atinge o consumo recomendado. O Brasil apresentou, neste período, um consumo médio de 153,3g/dia, correspondendo a $2,4 \%$ do total das calorias consumidas diariamente, permanecendo distante do mínimo recomendado, isso considerando apenas o ingerido nos domicílios (IBGE, 2010b). Os europeus consumiram, neste mesmo período, mais que o triplo da média brasileira, representando em média, 584g/dia. Nos Estados Unidos, o consumo médio per capita também é maior que o dos brasileiros, 392g/dia (USDA, 2010). No Brasil o consumo médio anual de F\&H, no período de 2008-09, foi respectivamente de $28,863 \mathrm{~kg}$ e $27,075 \mathrm{~kg}$. A aquisição domiciliar per capita desses alimentos, por regiões estão apresentadas na Figura 2.

Figura 2 - Aquisição alimentar domiciliar per capita anual (kg), por Grandes Regiões, segundo as frutas e hortaliças período 2008-2009 Fonte: Pesquisa de Orçamentos Familiares (POF) 2008-2009. IBGE. Aquisição de Alimentos Domiciliar, 2010.

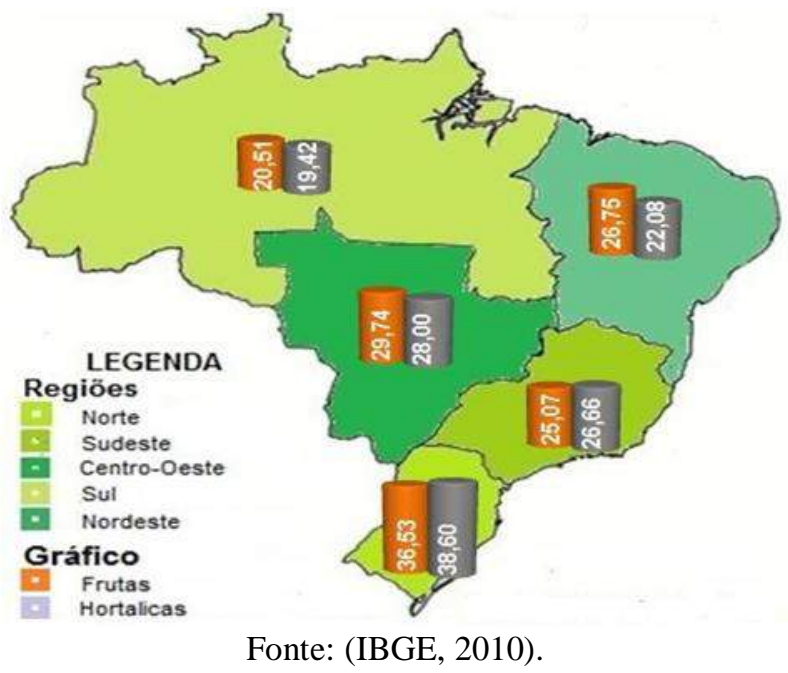

Quanto ao consumo médio por regiões, exposto na Figura 2, observa-se que a Região Sul possui médias de consumo acima da média nacional e das outras regiões, a qual apresentou respectivamente $36,531 \mathrm{~kg}$ pra frutas e $38,595 \mathrm{~kg}$ para hortaliças. Em contrapartida, a Região Norte apresenta as menores médias de consumo, apresentando respectivamente $20,511 \mathrm{~kg}$ e 19,418kg, ambas abaixo da média nacional e das demais regiões.

Referente ao consumo de $\mathrm{F} \& \mathrm{H}$ fora do lar, segundo pesquisa realizada pela Confederação Nacional de Agricultura - CNA, os percentuais caem em relação ao consumo domiciliar. Dos 1.420 entrevistados, 55\% não comem fora ou o faz raramente. Entre os que o fazem com regularidade, a média é de 8,8 vezes ao mês. Observou-se que quando comem fora, a inclusão de $\mathrm{F} \& \mathrm{H}$ na alimentação atinge percentuais bem inferiores àqueles relativos à alimentação no domicílio. Para as hortaliças estes percentuais de consumo fora e no domicílio são respectivamente de $44 \%$ e $69 \%$ e, para frutas, de $4 \%$ para $37 \%$. Assim, pode-se deduzir que quanto mais o indivíduo come fora, mas tende a reduzir o seu potencial de consumo de frutas e hortaliças (CNA, 2011). Isso acontece, em especial, com as frutas, já que a referida pesquisa destaca nas suas conclusões que, essas não possuem vínculo efetivo com as 
refeições, e que o seu consumo pode ser feito livremente durante o dia, isso a torna mais vulnerável ao esquecimento ou a substituições. Nesse contexto, é importante destacar que o Ministério da Saúde, por meio da Secretaria de Vigilância em Saúde em parceria com o Ministério de Educação - MEC e o IBGE, em 2009, realizou a I Pesquisa Nacional de Saúde do Escolar - PENSE, entre adolescentes de $9^{\text {a }}$ ano do ensino fundamental nos municípios das capitais e no Distrito Federal do país, tendo como um dos objetivos a verificação da alimentação no ambiente escolar. A pesquisa revelou que o consumo de guloseimas, pelos adolescentes, é superior ao consumo de frutas, em todas as regiões brasileiras. Dados da pesquisa indicaram que a região Sudeste e Sul apresentam o maior percentual de consumo de guloseimas, entre os adolescentes pesquisados e, isso corrobora com as estimativas da POF 200809, em que a prevalência, em percentual de obesidade no Brasil, na faixa etária de 10 a 19 anos, é maior nestas regiões.
Influência da renda na aquisição de frutas e hortaliças e na prevalência de obesidade

Mesmo separando os dados por região, verificou-se que o consumo de F\&H está abaixo do recomendado pela OMS. Para melhor entender as razões desse déficit de consumo, utilizou-se o Teste de Correlação de Pearson para identificar a existência de associações entre a variável renda e a quantidade consumida destes produtos, considerando as 27 unidades da federação. $O$ resultado do teste indicou a existência de uma correção positiva significativa $(\mathrm{r}=0,58811$ e $\mathrm{p}$-valor $=0,0013)$ entre as variáveis estudadas. Assim, pode-se dizer que os aumentos na variável renda estão associados com aumentos no consumo de $\mathrm{F} \& \mathrm{H}$, isto é, à medida que a renda aumenta, o consumo de F\&H também aumenta. Essa relação pode ser observada nos resultados da POF 2002-03 e 2008-09, os quais estão apresentados na Tabela 2.

Tabela 2 - Aquisição alimentar domiciliar per capita anual, por classes de rendimento total e variação patrimonial mensal familiar, segundo os produtos selecionados Brasil - período 2002-2003 e 2008-2009.

\begin{tabular}{|c|c|c|c|c|c|c|c|}
\hline \multicolumn{8}{|c|}{ Aquisição alimentar domiciliar per capita anual (kg) } \\
\hline \multirow[t]{2}{*}{ Produto } & \multirow[t]{2}{*}{$\begin{array}{l}\text { Média de } \\
\text { consumo/ano }\end{array}$} & \multicolumn{6}{|c|}{$\begin{array}{l}\text { Classes de rendimento total e variação patrimonial mensal } \\
\text { familiar }(R \$)\end{array}$} \\
\hline & & $\begin{array}{c}\text { Até } 830 \\
\text { (1) }\end{array}$ & $\begin{array}{c}\text { Mais de } \\
830 \text { a } \\
1245\end{array}$ & $\begin{array}{c}\text { Mais de } \\
1245 \text { a } \\
2490\end{array}$ & $\begin{array}{c}\text { Mais de } \\
2490 \text { a } \\
4150\end{array}$ & $\begin{array}{c}\text { Mais de } \\
4150 \text { a } \\
6225\end{array}$ & $\begin{array}{c}\text { Mais de } \\
6225\end{array}$ \\
\hline Frutas & 28,863 & 14,252 & 20,408 & 27,191 & 35,797 & 41,13 & 59,297 \\
\hline Hortaliças & 27,075 & 15,413 & 22,623 & 27,059 & 32,644 & 35,147 & 44,282 \\
\hline Total & 55,938 & 29,665 & 43,031 & 54,25 & 68,441 & 76,281 & 103,579 \\
\hline $\begin{array}{l}\text { Consumo } \\
\text { Médio/dia }\end{array}$ & 0,153 & 0,081 & 0,118 & 0,149 & 0,188 & 0,209 & 0,284 \\
\hline
\end{tabular}

Fonte: Pesquisa de Orçamentos Familiares (POF) 2008-2009. IBGE. Aquisição de Alimentos Domiciliar, 2010.

Nota: (1) Inclusive sem rendimentos.

Reportando-se à Tabela 2 verificou-se que a renda é um dos aspectos limitantes para a expansão do consumo de $\mathrm{F} \& \mathrm{H}$, principalmente das frutas. Assim, a renda é um dos elementos do ambiente social que influencia no padrão alimentar dos indivíduos (MACINTYRE et al., 2002; YEN \& SYME, 1999). Verificou-se que nos dois períodos das POFs, a diferença de consumo de $\mathrm{F} \& \mathrm{H}$ entre a menor renda e a maior é em torno de 239\%. Mesmo ainda não atingindo o recomendado, comparando o consumo de $\mathrm{F} \& \mathrm{H}$ da classe baixa e alta nos anos de 2002-03 e 2008-09, verificamos que referente às frutas o aumento da classe baixa e alta, foi de respectivamente $28,86 \%$ e $29,30 \%$. Quanto as hortaliças, as classes baixa e alta apresentaram incremento respectivamente de $1,71 \%$ e $4,66 \%$. Também observou-se que no grupo das frutas a diferença de consumo entre a menor e a maior renda no período de 2002-03 é de $314 \%$ e 
referente as hortaliças a diferença é de $169 \%$. No período de 2008-09 essa diferença é de $316 \%$ para as frutas e $187 \%$ para as hortaliças. Contudo, mesmo nas classes de alto poder aquisitivo, ainda há muito a ser explorado pela cadeia de produção desses produtos. Assim, baseado no baixo consumo per capita, destacamos que o setor de frutas e hortaliças possui um potencial de crescimento promissor. A luz das reflexões expostas acima sobre a influência da renda no consumo de $\mathrm{F} \& \mathrm{H}$ lembramos que os países desenvolvidos, apresentam consumo médio, desses produtos, superiores ao do Brasil, isso está atrelado a fatores como renda e hábitos alimentares. Além disso, existem na Europa e nos Estados Unidos iniciativas tanto do governo quanto da cadeia de produção que incentivam o consumo desses produtos (JULIÃO et al., 2010). No entanto, esses países, em especial os Estados Unidos estão liderando o ranking de obesidade. Isso nos remete a refletir sobre a relação do consumo desses produtos e a obesidade. Segundo a nutricionista Zanolla, mesmo as F\&H sendo consideradas como alimentos saudáveis devem ser ingeridas dentro de uma dieta balanceada, para que se mantenha ou obtenha um peso saudável ${ }^{6}$. Portanto, o consumo adequado desses alimentos auxilia na prevenção e no combate da obesidade, devido ao seu alto valor nutritivo e o baixo teor calórico (NATIONAL HEART FORUM, 1997a, 1997b; WCRF, 2007). Além disso, contribui para a diminuição do consumo de alimentos ricos em gordura e açúcar. Outro aspecto destacado pela nutricionista foi o aumento da prevalência da obesidade nas crianças das classes de alto poder aquisitivo, situação esta que a décadas atrás era mais acentuada nas famílias de baixa renda (informação verbal) ${ }^{7}$. Podemos constatar essa reflexão nos dados apresentados na Tabela 3.

Tabela 3- Prevalência de Obesidade na População com 5 a 9 anos de idade, 10 a 19 e 20 e mais idade de acordo com os critérios da Organização Mundial de Saúde - OMS, por sexo, segundo as classes de rendimento total e a variação patrimonial mensal familiar per capita - Brasil - período 2008-2009.

\begin{tabular}{|c|c|c|c|c|c|c|}
\hline \multirow{4}{*}{$\begin{array}{c}\text { Classes de } \\
\text { rendimento total } \\
\text { e variação } \\
\text { patrimonial } \\
\text { mensal familiar } \\
\text { per capita } \\
\text { (salários } \\
\text { mínimos) }\end{array}$} & \multicolumn{6}{|c|}{$\begin{array}{c}\text { Prevalência de obesidade na população brasileira por idade e } \\
\text { sexo }(\%)\end{array}$} \\
\hline & \multicolumn{6}{|c|}{ Faixa Etária } \\
\hline & \multicolumn{2}{|c|}{5 a 9} & \multicolumn{2}{|c|}{10 a 19} & \multicolumn{2}{|c|}{20 a mais idade } \\
\hline & $\begin{array}{l}\text { Masc } \\
\text { ulino }\end{array}$ & $\begin{array}{l}\text { Femini } \\
\text { no }\end{array}$ & $\begin{array}{c}\text { Masculin } \\
\mathbf{0}\end{array}$ & $\begin{array}{l}\text { Feminin } \\
\quad \text { o }\end{array}$ & $\begin{array}{l}\text { Masculin } \\
\quad \text { o }\end{array}$ & $\begin{array}{l}\text { Feminin } \\
\quad \text { o }\end{array}$ \\
\hline Até 1/4 & 11,8 & 7,4 & 1,8 & 1,9 & 5,5 & 15,1 \\
\hline $\begin{array}{l}\text { Mais de } 1 / 4 \text { a } \\
1 / 2\end{array}$ & 12,9 & 10,0 & 3,1 & 4,2 & 6,9 & 14,6 \\
\hline Mais de $1 / 2$ a 1 & 15,4 & 12,7 & 5,0 & 4,1 & 9,6 & 16,3 \\
\hline Mais de 1 a 2 & 19,5 & 13,8 & 9,2 & 4,6 & 13,3 & 18,0 \\
\hline Mais de 2 a 5 & 23,8 & 13,9 & 8,2 & 4,6 & 16,1 & 18,1 \\
\hline Mais de 5 & 30,8 & 14,8 & 8,2 & 2,6 & 17,1 & 15,8 \\
\hline
\end{tabular}

Fonte: Elaborado a partir dos dados da POF 2008-2009 IBGE. Antropometria e Estado Nutricional de Crianças, Adolescentes e Adultos no Brasil, 2010.

Observou-se que em todas as faixas etárias masculinas houve aumento das prevalências de obesidade e, a razão de prevalências entre classes extremas de renda oscilou aproximadamente de duas a cinco vezes.

\footnotetext{
${ }^{6}$ De acordo com o Ministério da Saúde, corresponde ao Índice de Massa Corporal ( IMC) de 18,5 a 24,9.
}

Já referente ao sexo feminino, na faixa de 5 a 9 anos de idade, as prevalências de obesidade aumentaram com a renda, e a razão de prevalências entre as classes extremas de renda, oscilou em torno de três vezes. No entanto, as

\footnotetext{
${ }^{7}$ ZANOLLA, Anelise. Entrevista concedida em 04.09.12. Porto Alegre. Consulta Médica.
} 
faixas etárias entre 10 e 19 anos e acima de 20 anos, as relações entre obesidade e renda familiar apresentaram curvilíneas, sendo as maiores prevalências observadas nas classes intermediárias de renda (IBGE, 2010a). Verificou-se que na faixa etária de 10 a 19 anos, do sexo masculino, ocorre a maior oscilação, entre as classes extremas de renda. Essa informação corrobora com o resultado da pesquisa do PENSE, o qual já apresentado anteriormente (Figura 3). Em que jovens nesta faixa etária, em todas as regiões brasileira, estão preferindo mais guloseimas a frutas, tanto nas escolas públicas como privadas. Talvez essas escolhas estejam atreladas não só ao preço, mas também as preferências dos adolescentes por alimentos não saudáveis. Estudos confirmam que, o consumidor tem aumentado a preferência por produtos alimentares com alta densidade energética $e$, que geralmente apresentam elevado teor de carboidratos simples e gorduras (BOOTH et al., 2005).

Ações de estímulo ao consumo de frutas e hortaliças no Brasil, nas dimensões micro e macroambiente que contribuem para a intervenção da obesidade

Um modelo conceitual para identificar e priorizar intervenções ambientais para prevenir e combater a obesidade foi proposto por Swinburn, Egger e Raza (1999), o qual os autores denominaram Analysis Grid for Environmental Linked to Obesity - ANGELO. Neste modelo o ambiente é dividido em relação à dimensão (micro ou macro) e ao tipo (física, econômica, política e sociocultural). De acordo com essa proposta de divisão o macroambiente seria aquele que influencia toda a população e o microambiente seria composto por locais mais próximos dos indivíduos, como as escolas e a vizinhança, no caso das crianças; e o local de trabalho e a vizinhança, no caso dos adultos (SWINBURN et al., 1999). Nesse contexto, as políticas e os programas voltados para a prevenção e o controle do ganho de peso devem incentivar mudanças no âmbito individual, modificações na sociedade e no ambiente onde as pessoas vivem e trabalham, para que sejam realmente eficazes na redução da obesidade nas diversas populações (WHO, 2002). Com o intuito de atender essas recomendações, no Brasil, em 2006, foi aprovada a Política Nacional de Promoção da Saúde, a qual prioriza entre outras ações a alimentação saudável e a atividade física, com transferência de recursos federais a estados e municípios para a implantação dessas ações de uma forma intersetorial e integrada (BRASIL, 2011b). Referente à alimentação saudável, a Política Nacional de Promoção à Saúde visa fomentar políticas públicas que garantam o acesso a esta, considerando as especificidades culturais, regionais e locais, através da promoção da articulação e mobilização dos setores público e privado para a adoção de ambientes que favoreçam a alimentação saudável. Também é objetivo desta Política estimular o desenvolvimento de campanhas publicitárias para orientar e sensibilizar a população sobre os benefícios desse tipo de alimentação, tendo como referência o Guia Alimentar para a População Brasileira (BRASIL, 2006). O referido Guia foi editado pelo Ministério da Saúde com o objetivo de servir como base para as políticas públicas de alimentação saudável. Este Guia, entre outras indicações, recomenda que as crianças, adolescentes e jovens tenham prioridade em programas governamentais de alimentação saudável e, além disso, que frutas e hortaliças devam ser inseridas nas refeições principais e lanches dos brasileiros (BRASIL, 2005). Referente ao consumo de frutas e hortaliças o Guia segue as diretrizes da Política Nacional de Alimentação e Nutrição- PNAN, a qual possui entre as suas prioridades estimular o consumo desses alimentos no País, cujos objetivos estão relacionados ao controle e prevenção das doenças nutricionais e das doenças crônicas não transmissíveis, responsáveis por $70 \%$ dos gastos do Sistema Único de Saúde-SUS (BRASIL, 2011b).

Considerando estas orientações e ciente das crescentes taxas de obesidade das crianças de 5a 9 anos, e dos adolescentes e jovens de 10 a 19 anos, o governo brasileiro decidiu ampliar o Programa Nacional de Alimentação Escolar (PNAE), através da Portaria Interministerial $\mathrm{n}^{\circ}$ 1.010, de 08 de maio de 2006, a qual reforça a importância do consumo de frutas e hortaliças 
no ambiente escolar. Essa Portaria instituiu diretrizes para a promoção da alimentação saudável nas escolas de educação infantil, ensino fundamental e médio das redes pública e privada no Brasil. Um dos pontos de destaque dessa Portaria é aumentar a oferta e promover o consumo de $\mathrm{F} \& \mathrm{H}$, incorporando todas as recomendações nutricionais em projetos pedagógicos com viés em educação nutricional (BRASIL, 2006). Ressalta-se que todos os alimentos ofertados nas escolas passaram por um teste de aceitabilidade, que corresponde a aceitação no cardápio por um determinado período de tempo, com exceção das $\mathrm{F} \& \mathrm{H}$, por entenderem que estes são alimentos essenciais para a saúde. O Fundo Nacional de Desenvolvimento da Educação - FNDE, recomenda através da resolução no 38 , de 16 de julho de 2009, que o cardápio escolar inclua pelo menos $200 \mathrm{~g}$ de $\mathrm{F} \& \mathrm{H}$ por semana por aluno da rede pública, representando 40g/dia. Essa quantidade corresponde à metade da recomendação da OMS e do Guia Alimentar para a População Brasileira. O número de alunos matriculados, na rede pública, em 2010, atendido pelo PNAE, corresponde a 47 milhões de alunos, no entanto apenas $40 \%$ das escolas, neste mesmo período, implementaram o recomendado pelo FNDE (BRASIL, 2010c). A luz do que foi exposto, verificamos que através da oferta de alimentos saudáveis nas escolas públicas, e recomendando o consumo desses nas privadas, o governo brasileiro, através de políticas públicas, vem buscando contribuir para que este ambiente seja mais saudável. Um ambiente saudável é definido como um ambiente que incentiva as escolhas saudáveis, este é caracterizado como leptogênico, o oposto do obesogênico, e por isso responsável por prevenir e combater a obesidade. Essa ação no âmbito das escolas, como por exemplo, o Programa mais Fruta na escola, contribui para a promoção de um ambiente leptogênico, por sua vez, é caracterizada de dimensão microambiental. (SWINBURN et al., 1999). Também ações macroambientais vêm sendo desenvolvidas no País, tais como: O Programa Saborosa Brincadeira e 5 ao dia. O Quadro I, apresenta algumas ações de estímulo ao consumo de frutas e hortaliças, de dimensão micro e macroambiental.

Quadro I - Iniciativas de Estímulo ao Consumo de Frutas e Hortaliças Desenvolvidas no Brasil

\begin{tabular}{|c|c|c|c|c|}
\hline PROGRAMA & $\begin{array}{l}\text { Merenda Escolar } \\
\text { (Dimensão } \\
\text { Microambiental) }\end{array}$ & $\begin{array}{c}\text { Mais Fruta na } \\
\text { Escola } \\
\text { (Dimensão } \\
\text { Microambiental) }\end{array}$ & $\begin{array}{c}5 \text { ao Dia } \\
\text { (Dimensão } \\
\text { Macroambiental) }\end{array}$ & $\begin{array}{c}\text { Saborosa } \\
\text { Brincadeira } \\
\text { (Dimensão } \\
\text { Macroambiental) }\end{array}$ \\
\hline ORGANIZAÇÃO & $\begin{array}{lr}\text { PNAE } & \text { (Programa } \\
\text { Nacional } & \text { de } \\
\text { Alimentação } & \text { Escolar } \\
\text { e FNDE } & \text { (Fundo } \\
\text { Nacional } & \text { de } \\
\text { Desenvolvimento } \\
\text { Nacional. }\end{array}$ & $\begin{array}{l}\text { ABPM (Associação } \\
\text { Brasileira de } \\
\text { Produtores de } \\
\text { Maças) e Secretaria } \\
\text { de Educação de } \\
\text { Santa Catarina. }\end{array}$ & $\begin{array}{lr}\text { IBRA } & \text { (Instituto } \\
\text { Brasileiro } & \text { de } \\
\text { Orientação } & \\
\text { Alimentar). } & \end{array}$ & $\begin{array}{l}\text { IBRAF (Instituto } \\
\text { Brasileiro de Frutas e } \\
\text { Hortaliças), SEBRAE } \\
\text { (Serviço Brasileiro de } \\
\text { Apoio às Micro e } \\
\text { Pequenas Empresas) e } \\
\text { Benassi. }\end{array}$ \\
\hline PROL & Frutas e Hortaliças & Maçã & Frutas e Hortaliças & $\begin{array}{c}\text { Frutas (uva, caqui, } \\
\text { limão, manga, } \\
\text { abacaxi, figo, acerola, } \\
\text { goiaba pêssego, } \\
\text { ameixa e nêspera). }\end{array}$ \\
\hline PROBLEMA & $\begin{array}{l}\text { Baixo consumo de } \\
\text { frutas e hortaliças. }\end{array}$ & $\begin{array}{l}\text { Baixo consumo de } \\
\text { frutas. }\end{array}$ & $\begin{array}{l}\text { Baixo consumo de } \\
\text { frutas e hortaliças }\end{array}$ & $\begin{array}{l}\text { Baixo consumo de } \\
\text { frutas. }\end{array}$ \\
\hline ESTRATÉGIA & $\begin{array}{l}\text { Oferta de frutas e } \\
\text { hortaliças nas escolas } \\
\text { públicas. }\end{array}$ & $\begin{array}{lr}\text { Distribuição } & \mathrm{de} \\
\text { maçãs } & \mathrm{e} \\
\text { informativos } & \mathrm{em} \\
\text { escolas. } & \end{array}$ & $\begin{array}{l}\text { Propõe o consumo de } \\
5 \text { porções de frutas e } \\
\text { hortaliças por dia. } \\
\text { Entre as estratégias } \\
\text { estão às promoções de } \\
\text { palestras } \\
\text { seminários. }\end{array}$ & $\begin{array}{l}\text { Degustação de frutas } \\
\text { de maneira divertida } \\
\text { no supermercado. }\end{array}$ \\
\hline
\end{tabular}


(MELO et al., 2016)

\begin{tabular}{|c|c|c|c|c|}
\hline PÚBLICO-ALVO & $\begin{array}{l}\text { Crianças da pré- } \\
\text { escola até o ensino } \\
\text { médio das escolas } \\
\text { públicas de todo } \\
\text { Brasil. Incluindo } \\
\text { modalidades voltadas } \\
\text { a jovens e adultos } \\
\text { (EJA). }\end{array}$ & $\begin{array}{l}\text { Crianças da pré- } \\
\text { escola } \\
\text { até a } 8^{\text {a }} \text { série de } \\
\text { Santa Catarina. }\end{array}$ & População em geral & $\begin{array}{l}\text { Crianças de } 2 \text { a } 5 \text { anos } \\
\text { do estado de São } \\
\text { Paulo }\end{array}$ \\
\hline $\begin{array}{l}\text { SITUAÇÃO } \\
\text { ATUAL } \\
\text { (até o momento da } \\
\text { pesquisa) }\end{array}$ & $\begin{array}{l}\text { Em andamento } \\
\text { Atende a } 40 \% \text { das } \\
\text { escolas públicas. }\end{array}$ & Extinto em 2010. & $\begin{array}{l}\text { Essas ações têm tido } \\
\text { pouco alcance no } \\
\text { País. }\end{array}$ & $\begin{array}{l}\text { Parado por falta de } \\
\text { investimento. }\end{array}$ \\
\hline
\end{tabular}

Fonte: Instituto Brasileiro de Frutas (IBRAF), 2012, Revista HortiFruti Brasil, 2010 e Ministério da Educação (2012).

Os Programas Saborosa Brincadeira e 5 ao dia, utilizaram uma estratégia de marketing que é bastante difundida nos Estados Unidos, o generic advertising, este representa o marketing voltado para os atributos do produto e não para a marca. O principal objetivo desses Programas foi aumentar a demanda total pelo produto, visando criar um hábito de consumo de frutas e, assim, beneficiar todos os atuantes da cadeia de produção. Além disso, contribuir para a redução da obesidade no Brasil, baseando-se nas recomendações do Ministério da Saúde. As suas atividades de campanhas publicitárias focaram nas características comuns das frutas, no caso destacando o valor nutricional, o qual, comumente, não é facilmente percebido pelos consumidores, em especial o público infantil. Para a realização dessas campanhas, foi necessária a cooperação entre os produtores envolvidos nos Programas.

Projeções de obesidade e de consumo de frutas e hortaliças no Brasil
O Governo Brasileiro visando ampliar suas ações de intervenção na área da saúde formulou um Plano de Ações Estratégicas para o Enfrentamento das Doenças Crônicas não Transmissíveis-DCNT, para período de 2011 a 2022. Este Plano tem como objetivo desenvolver ações em nível coletivo, para favorecer escolhas saudáveis no nível individual, promover o desenvolvimento e a implementação de políticas públicas efetivas, integradas, sustentáveis e baseadas em evidências para a prevenção e o controle das DCNT e seus fatores de risco e fortalecer articulações intersetoriais que promovam a construção de estilos de vida saudáveis (BRASIL, 2011b). Entre as estratégias estabelecidas estão: ampliar e fortalecer as ações de alimentação saudável e articular ações para a prevenção e combate a obesidade. Para implementar essas ações, o governo brasileiro também, em seus planos de intervenção para a obesidade, contempla estratégias para o setor produtivo. A Figura 4 apresenta as medidas para o setor produtivo de frutas e hortaliças (BRASIL, 2011b). 
Figura 4 - Principais Ações do Plano de DCNT em Promoção da Saúde: alimentação saudável, voltadas para o Agronegócio de Frutas \& Hortaliças.

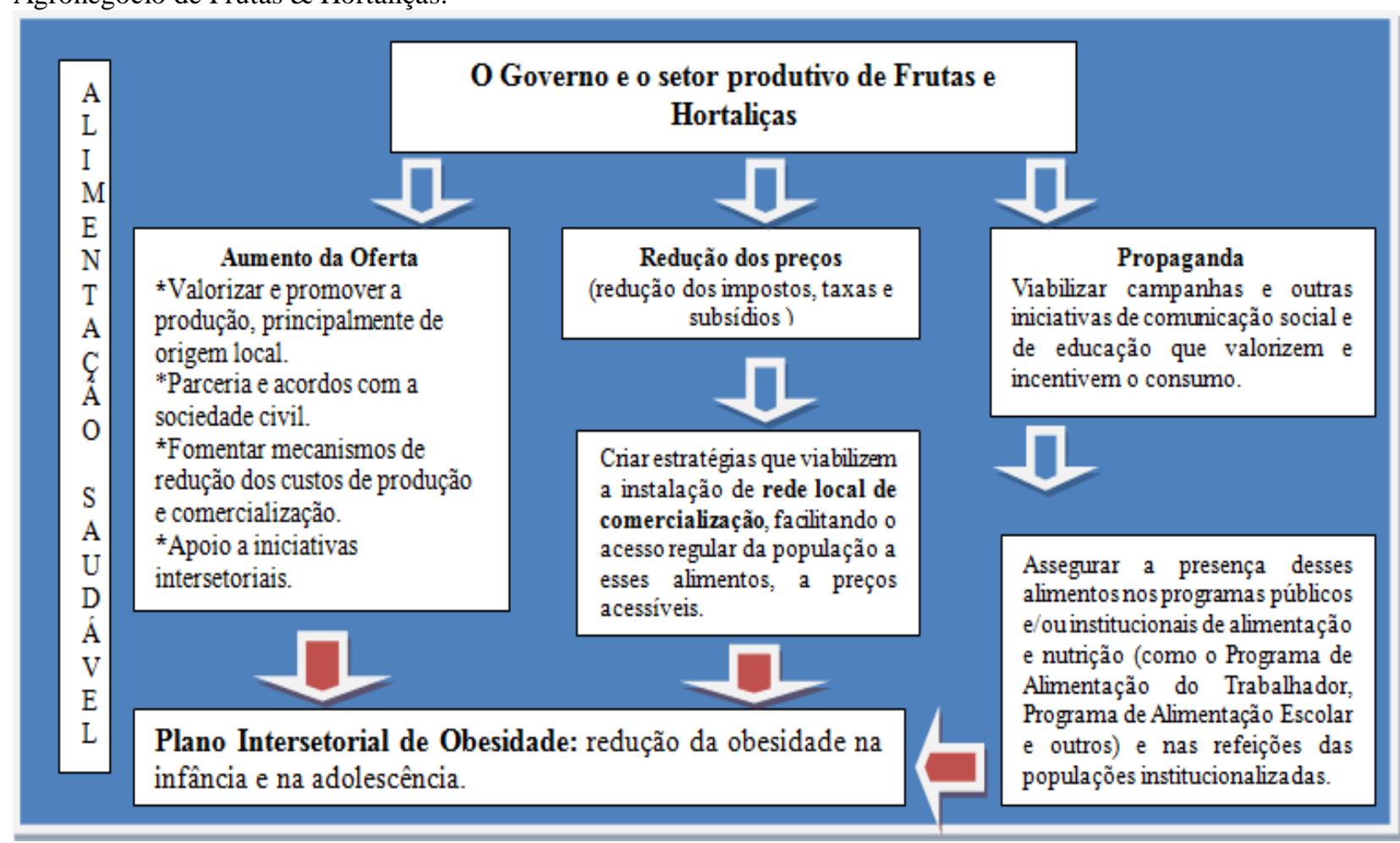

Fonte: Elaborado a partir do Plano de Ações Estratégicas para o Enfrentamento da DCNT no Brasil 2011-2022.

Como exposto na figura acima, há um esforço por parte do governo em estimular o consumo de $\mathrm{F} \& \mathrm{H}$, em especial, na fase infantil. Isso considerando que, estratégias de prevenção e controle do peso corporal podem ser mais efetivas quando desenvolvidas durante a infância (DIEZTZ, 1993; EPSTEIN et al., 1994). Ressaltamos que, uma das metas estabelecidas no Plano é ampliar e fortalecer o PNAE, visando ofertar esses produtos, até o final de 2022, a $80 \%$ das escolas públicas. A meta é reduzir até 2022 em $50 \%$ as taxas de obesidade entre crianças de 5 a 9 anos e jovens de 10 a 19 anos (BRASIL, 2011b). Caso as metas sejam atingidas e atendendo as recomendações oficiais de consumo (80g/dia/aluno), estimando-se 47 milhões de alunos matriculados na rede pública e considerando que, o consumo anual por aluno, durante o ano letivo de 250 dias, o consumo de F\&H deverá ser de $20 \mathrm{~kg}$ de frutas e hortaliças/ ano/aluno, ou seja, 940 mil de toneladas por ano letivo/aluno poderá alavancar 47 mil hectares de frutas e hortaliças (BRAGA \& BANZATO, 2010). Mediante o apresentado, analisando apenas a alimentação escolar, sugerimos que o aumento de consumo pode ser uma oportunidade para o agronegócio frutícola e olerícola do Brasil.

\section{Conclusões}

Esse estudo partiu da premissa de que o consumo de frutas e hortaliças contribui para a prevenção e o combate a obesidade. Daí a importância de analisar o consumo desses alimentos no Brasil, identificar a influência da renda nesse consumo e verificar quais as ações desenvolvidas para estimular o consumo desses alimentos. A pesquisa apontou que, o consumo de F\&H está abaixo do recomendado pela OMS, e que um dos fatores que contribuem para esse déficit de consumo é a renda per capita. Considerando que a maioria da população brasileira possui renda baixa, sugere-se um maior apoio governamental, através de políticas públicas para estimular o consumo desses produtos. Quanto às ações de estímulo ao consumo de frutas e hortaliças, ainda são escassas no Brasil. Além disso, quando são colocadas em prática, logo deixam de existir por falta de investimento. Por outro lado, verificamos que há espaço para crescimento das 
vendas de $\mathrm{F} \& \mathrm{H}$ no mercado interno, pois como já referido, o consumo desses produtos ainda é baixo e, assim, apresenta possibilidade de ganhos para toda a cadeia de produção. Verificou-se, também, que são poucas as iniciativas de estímulo ao consumo de $\mathrm{F} \& \mathrm{H}$, por parte dos produtores brasileiros no âmbito macroambiental, talvez eles não se vejam como responsáveis pelo marketing de seus produtos. Uma alternativa de publicidade pode ser o generic advertising, em que os custos serão diluídos entre os produtores participantes da estratégia. Já em países desenvolvidos, como nos Estados Unidos, são várias as iniciativas de incentivo ao consumo que partem dos próprios produtores. A diferença é que, nesses países, a cadeia já tem estabelecida junto aos governos uma dinâmica que permite o direcionamento de parte da renda gerada com a venda do produto para investimentos de marketing (JULIÃO et al., 2010). É importante que esse modelo de alocação de receita também seja estabelecido no Brasil a fim de serem gerados fundos de desenvolvimento do marketing para o consumo de F\&H.

Outro aspecto a ser considerado é o foco das campanhas existentes. A maior parte dos

\section{Referências}

BARDIN, L. Análise de Conteúdo. Lisboa: Edições 70, 1977.

BOOTH, K. M.; PINKSTON, M. M.; POSTON, W. S. Obesity and the built environment. J Am Diet Assoc. 2005 May;105(5 Suppl 1): S110-7.

BRAGA, D.; BANZATO, T. C. Alimentação escolar: merenda é oportunidade para o hortifrutiultor. São Paulo, ano 8, No. 89, abr. 2010.

BRASIL. Guia alimentar para a população brasileira: promovendo a alimentação saudável. Ministério da Saúde. Secretaria de Atenção à Saúde. Brasília: Ministério da Saúde, 210 p. (Série A. Normas e Manuais Técnicos). 2005. investimentos para o aumento do consumo de $\mathrm{F} \& \mathrm{H}$ tem como mensagem principal a questão da saudabilidade. Com isso a imagem desses produtos, fica muito atrelada ao uso medicinal visando a cura de alguma enfermidade, condicionando seu consumo a ocorrência de doenças. Sugere-se que a imagem das F\&H seja atrelada como parte das refeições diárias, devido seu caráter funcional na alimentação. Quanto à ação do governo frente à alimentação escolar, este vem ampliando suas ações, visando até 2022 atingir a $80 \%$ das escolas públicas do País. No passado, a preocupação era a desnutrição e hoje se discute o papel da escola também como educadora alimentar, inserindo conceitos de alimentação saudável e combatendo a obesidade. No entanto, também é preciso expandir as ações desenvolvidas nas escolas privadas, por exemplo, acompanhamento do que está sendo ofertado nas cantinas dessas escolas.

O estudo, ainda, apontou a importância do agronegócio em ofertar frutas e hortaliças, considerando as recomendações da Estratégia Global para Alimentação Saudável da OMS, e para estimular esta oferta o governo brasileiro tem como uma das estratégias incentivar o crescimento do setor frutícola e olerícola.

BRASIL. Ministério da Saúde. Secretaria de Vigilância em Saúde. Secretaria de Gestão Estratégica e Participativa. Vigitel Brasil 2010. Vigilância de fatores de risco e proteção para doenças crônicas por inquérito telefônico. Brasília: Ministério da Saúde, 152 p. (Série G. Estatística e Informação em Saúde). 2011a.

BRASIL. Ministério da Saúde. Secretaria de Vigilância em Saúde. Departamento de Análise de Situação de Saúde. Plano de ações estratégicas para o enfrentamento das doenças crônicas não transmissíveis (DCNT) no Brasil 2011-2022. Brasília: Ministério da Saúde. 2011b.

BRASIL. Ministério da Educação. Fundo Nacional de Desenvolvimento da Educação. Programa de alimentação Escolar. Brasília: Ministério da Educação. 2011c. Disponível em: $\quad$ http://www.fnde.gov.br/index.php/aeapresentacao. Acesso em: 30 ago. 2012. 
BRASIL. Portaria Interministerial $n^{0} 1.010$ de, 08 de maio de 2006. 2006. Disponível em: http://dtr2001.saude.gov.br/sas/PORTARIAS/P ort2006/GM/GM-1010.htm. Acesso em: 30 ago. 2012.

Confederação Nacional de Agricultura. Pesquisa Quantitativa: Consumo de fruta e hortaliças. Connection Research. 2011. Disponível em: http://www.agricultura.gov.br/arq_editor/file/.../ Hortalicas/25.../Promocao_Consumo.pdf

Acesso em: 26 ago. 2012.

CYPRESS, M. Looking upstream. Diabetes Spectrum. Vol. 17, 2004, pp. 249-253.

DIETZ, W. H. Therapeutic strategies in childhood obesity. Horm Res, pp. 1993; 39, Suppl 3:86- 90.

EPSTEIN L.H., WING R.R., VALOSKI A. Childhood obesity. Pediatr Clin North Am Journal of the American Dietetic Association, New York, Vol. 105, No. 5, pp. 110S-117S, Supplementum 1. 2005.

FAO/WHO/ONU. Human energy requirements. Report of a Joint FAO/WHO/UNU Expert Consultation. FAO Food and Nutrition Technical Report Series No. 1. Rome: Food and Agriculture Organization. 2004.

Instituto Brasileiro de Geografia e Estatística (IBGE). Pesquisa Nacional de Saúde do Escolar (PENSE). 2009. Disponível em: http://www.ibge.gov.br/home/estatistica/popula cao/condicaodevida/. Acesso em: 28 ago. 2012.

Instituto Brasileiro de Geografia e Estatística (IBGE). Estudo Nacional da Despesa Familiar (Endef) 1974-1975. 1976. Disponível em: http://www.ibge.gov.br/home/estatistica/popula cao/condicaodevida/. Acesso em: 28 ago. 2012.

Instituto Brasileiro de Geografia e Estatística (IBGE). Pesquisa de Orçamentos Familiares (POF) 2002-2003. Antropometria e estado nutricional de crianças, adolescentes e adultos no Brasil. Rio de Janeiro: IBGE, 2004a.
Instituto Brasileiro de Geografia e Estatística (IBGE). Pesquisa de Orçamentos Familiares (POF) 2002-2003, Aquisição de alimentos Domiciliar per capita: Brasil e grandes regiões. 2004b. Disponível em: http://www.ibge.gov.br/home/estatistica/popula cao/condicaodevida/. Acesso em: 23 ago. 2012.

Instituto Brasileiro de Geografia e Estatística (IBGE). Pesquisa de Orçamentos Familiares (POF) 2008-2009. Antropometria e estado nutricional de crianças, adolescentes e adultos no Brasil. Rio de Janeiro: IBGE, 2010a.

Instituto Brasileiro de Geografia e Estatística (IBGE). Pesquisa de Orçamentos Familiares (POF) 2008-2009, Aquisição de alimentos Domiciliar per capita: Brasil e grandes regiões. 2010b. Disponível em: http://www.ibge.gov.br/home/estatistica/popula cao/condicaodevida/. Acesso em: 25 ago. 2012.

JULIÃO, L. et al.; SÁBIO, R. P.; GALESKAS, H. Marketing de hortifrutis. HortiFruti Brasil. São Paulo, ano 10, No. 109, pp. 6-10, jan./fev. 2010.

LIU, S.; MANSON, J.E., LEE, I.M., COLE, S.R., HENNEKENS C.H., WILLETT W.C. et al. Fruit and vegetable intake and risk of cardiovascular disease: the Women's Health Study. Am J Clin Nutr. Vol. 72, No. 4, pp. 9228. 2000.

MACINTYRE, S., ELLAWAY, A., CUMMINS, S. Place effects on health: how can we conceptualise, operationalise, and measure them? Soc Sci Med, Vol. 55, pp. 125-139. 2002.

MALTA, D. C.; MOURA, L.; SOUZA, M. F. et al. Lung cancer, cancer of the trachea, and bronchial cancer: mortality trends in Brazil, 1980-2003. J. Bras. Pneumol. No.33, pp. 53643. 2007.

NATIONAL HEART FORUM (UK). At least five a day: strategies to increase vegetable and fruit consumption. London: National Forum. 1997a. 
OECD. Health at a Glance 2011: OECD Indicators. 2011. Disponível em: http://dx.doi.org/10.1787/health_glance-2011. Acesso em: 25 ago. 2012.

POPKIN, B. M.; DOAK, C.M. The obesity epidemic is a wordwide phenomenon. Nutrition Review. Vol. 56, pp 106-114. 1998.

RIBOLI, E.; NORAT, T. Epidemiologic evidence of the protective effect of fruit and vegetables on cancer risk. American Journal of Clinical Nutrition, No. 78 (Supl.), pp. 559S569S. 2003.

SCHMIDT, M. I.; DUNCAN, B. B.; SILVA, G. A.; MENEZES, A. M.; MONTEIRO, C. A.; BARRETO, S. M.; CHOR, D.; MENEZES, P. R. Chronic non-communicable diseases in Brazil: burden and current challenges. The Lancet, No377. 2011.

Sociedade Brasileira De Cirurgia Bariátrica E Metabólica. Evolução da Cirurgia Bariátrica e Metabólica no Brasil. 2012. Em: http://www.sbcb.org.br/cbariatrica.asp?menu=0 . Acesso em: 30 ago. 2012.

SWINBURN, M.D.; EGGER, G.; RAZA, F. Dissecting Obesogenic Environments: The Development and Application of a Framework for Identifying and Prioritizing Environmental Interventions for Obesity. Preventive Medicine, Vol. 29, pp 563-570. 1999.
United States Department of Agriculture (USDA). National Nutrient Database for Standard Reference. Agricultural Research Service. EUA, No.72. 2010. Disponível em: http://www.unifesp.br/dis/servicos/nutri/.

Acesso em: 28 ago. 2012.

World Cancer Research Fund (WCRF). Food, nutrition and the prevention of cancer: a global perspective. Washington DC: AICR, 2007. Disponível em: http://eprints.ucl.ac.uk/4841/1/4841.pdf.

Acesso em: 29 ago. 2012.

World Health Organization. Obesity and overweight. What are overweight and obesity? WHO, 2011a. Disponível em: http://www.who.int/mediacentre/factsheets/fs31 1/en. Acesso em: 29 ago. 2012.

World Health Organization. The World health report 2002: Reducing risks, promoting healthy life. Geneva: WHO, 2002. Disponível em: http://www.who.int/mediacentre/factsheets/fs31 1/en/. Acesso em: 29 ago. 2012.

World Health Organization. Working to overcome the global impact of neglected tropical diseases. WHO, 2011b. Disponível em: http://www.who.int/mediacentre/factsheets/fs31 1/en. Acesso em: 29 ago. 2012. 